\title{
Endoscopic Scoring System for Gastric Atrophy and Intestinal Metaplasia: Correlation with OLGA and OLGIM Staging: A Single Center Prospective Pilot Study
}

\section{Hee Kyong $\mathrm{Na}$}

University of Ulsan College of Medicine, Asan Medical Center

Kee Don Choi ( $\square$ keedon@amc.seoul.kr)

University of Ulsan College of Medicine, Asan Medical Center

Young Soo Park

University of Ulsan College of Medicine, Asan Medical Center

Hwa Jung Kim

University of Ulsan College of Medicine, Asan Medical Center

Ji Yong Ahn

University of Ulsan College of Medicine, Asan Medical Center

Jeong Hoon Lee

University of Ulsan College of Medicine, Asan Medical Center

Kee Wook Jung

University of Ulsan College of Medicine, Asan Medical Center

Do Hoon Kim

University of Ulsan College of Medicine, Asan Medical Center

Ho June Song

University of Ulsan College of Medicine, Asan Medical Center

Gin Hyug Lee

University of Ulsan College of Medicine, Asan Medical Center

Hwoon-Yong Jung

University of Ulsan College of Medicine, Asan Medical Center

\section{Research Article}

Keywords: Gastritis, Atrophy, Metaplasia, Endoscopy, Narrow band imaging

Posted Date: October 15th, 2021

DOI: https://doi.org/10.21203/rs.3.rs-948227/v1 
License: (c) (i) This work is licensed under a Creative Commons Attribution 4.0 International License. Read Full License

Version of Record: A version of this preprint was published at Scandinavian Journal of Gastroenterology on April 6th, 2022. See the published version at https://doi.org/10.1080/00365521.2022.2055974. 


\section{Abstract}

Background/Aims: We aimed to develop an endoscopic scoring system to evaluate atrophic and intestinal metaplasia using narrow-band imaging (NBI) and near focus mode (NFM) to compare endoscopic scores with the Operative link for gastritis assessment (OLGA) and the Operative link for gastric intestinal metaplasia assessment (OLGIM).

Methods: A total of 51 patients who underwent diagnostic esophagogastroduodenoscopy were prospectively enrolled and endoscopic scoring using NBI and NFM was performed. Four areas (the lesser and greater curvatures of the antrum and the lesser and greater curvature side of the corpus) were observed and biopsies were taken. The degree of atrophy was scored from 0 to 2 according to the Kimura-Takemoto classification (0: $\mathrm{C} 0-1,1: \mathrm{C} 2-3,2: 01-3)$. The degree of metaplasia was scored from 0 to 4 (0: no metaplasia, 1: presence of metaplasia at the antrum, 2: presence of metaplasia at the corpus, add score 1: presence of metaplasia for 1/2> observed field of the picture at the antrum, add score 2: 1/2 $>$ observed field of the picture at the corpus). The endoscopic scores were compared to the OLGA and OLGIM staging.

Results: The correlation coefficient for atrophy between the endoscopic and histologic scores was 0.70 (95\% Cl: $0.52-0.81 p<0.001)$ and for metaplasia, it was 0.75 (95\% Cl: $0.60-0.85 ; p<0.001)$. For atrophic gastritis, endoscopic score $>1$ correlated with OLGA stage III and IV with a sensitivity, specificity, positive predictive value, negative predictive value, and agreement of $88 \%, 74 \%, 75 \%, 87 \%$, and $80.4 \%$, respectively, and for metaplasia, an endoscopic score $>1$ correlated with high OLGIM stage III and IV with $100 \%, 59 \%$, $69 \%, 100 \%$, and $78.4 \%$, respectively.

Conclusions: Endoscopic scoring for gastric atrophy and metaplasia using NBI-NFM correlate well with histologic staging.

\section{Introduction}

Atrophic gastritis and intestinal metaplasia are some of the first steps in the precancerous cascade for intestinal-type gastric cancer according to the Correa hypothesis[1]. In a nationwide cohort study in the Netherlands, the annual incidence of gastric cancer was reported as $0.1 \%$ for atrophic gastritis and $0.25 \%$ for intestinal metaplasia [2]. The extension and severity of the atrophy and intestinal metaplasia was significantly associated with an increased risk of intestinal-type gastric cancer [3-5].

The Operative Link for Gastritis Assessment (OLGA) system and the Operative Link for Intestinal Metaplasia (OLGIM) system were suggested for reporting the staging and grading of gastric atrophy and metaplasia according to the updated Sydney system classification [6, 7]. However, to apply the OLGA and OLGIM systems, multiple biopsies from at least four sites is mandatory, and they are therefore difficult to apply in a clinical setting because they are time-consuming and have a risk of bleeding. 
Diagnosis using narrow-band imaging (NBI) and magnification endoscopy (ME) for observing atrophy and intestinal metaplasia of the gastric mucosa has been reported [8-13]. The sensitivity and specificity of NBI-ME findings for atrophy and metaplasia were reported to be as high as $80-100 \%$. However, conventional magnifying endoscopy requires substantial skill and time, especially when operating at the zoom level.

Magnification using NFM has been recently introduced to enable 45-fold magnification on a 26-inch monitor under the control of a single button, which is easily applicable [14]. Studies using NBI with near focus mode have been reported, mainly for colon polyps [15-17]. As far as we know, there is no prior report using NBI with NFM in the stomach. Therefore, this study aimed to develop an endoscopic scoring system to evaluate atrophic gastritis and metaplasia using NBI and NFM and to compare the endoscopic scores with the histology-OLGA and OLGIM staging systems.

\section{Patients And Methods}

\section{Patients}

This study was a single-center, prospective pilot study. Between 2017 and 2019, consecutive patients who underwent diagnostic esophagogastroduodenoscopy (EGD) at Asan Medical Center, Seoul, Korea were enrolled. The following patients were excluded during screening: 1) patients who did not have an alert mentality, 2) patients who had unstable vital signs, 3) patients who were suspicious for gastrointestinal bleeding, 4) patients who were prescribed anticoagulant or antiplatelet agents and could not quit their medication, 5) patients who had undergone gastrectomy, 6) patients who had coagulopathy, and 6) patients who could not have multiple biopsies taken for any reason. Written informed consent was obtained from 61 patients, and 10 patients withdrew their consent. Finally, 51 patients were included in the study.

This study was performed following the Helsinki Declaration of the World Medical Association. This study was approved by the Institutional Review Board of Asan Medical Center (number: 2017-0947) and was registered with a clinical research information service (http://cris.nih.go.kr, KCT0004377).

\section{Endoscopic evaluation}

A conscious sedation with midazolam $(0.05-0.1 \mathrm{mg} / \mathrm{kg})$ was performed at the request of the patient. Upper endoscopy was performed using a white light endoscope with NBI and dual focus (standard and NFM) using GIF-HQ290 scope and EVIS-HQ290 (Olympus Medical System Co. Ltd, Tokyo, Japan). All endoscopic examinations were performed by two expert endoscopists (CKD and NHK). The gastric mucosa of the antrum and corpus were examined with white light endoscopy (WLE), NBI and NFM. Representative endoscopic pictures using WLE, NBI and NFM were taken at four sites: the lesser curvature of the antrum, the greater curvature of the antrum, the lesser curvature of the corpus and the greater curvature of the corpus, and video of the endoscopic examination was also taken. Endoscopic scoring for gastric atrophy and intestinal metaplasia was performed during the exam by a performing endoscopist. If 
there are ambiguous findings for scoring, it was scored after discussion of two endoscopists with the endoscopic images. Endoscopic exam using NBI and dual focus mode required additive several minutes compared to exam using white light endoscopy alone.

Biopsies were taken from the same four sites (the lesser curvature of the antrum, the greater curvature of the antrum, the lesser curvature of the corpus, and the greater curvature of the corpus). A rapid urease test (Hp Kit: Chongkungdang Pharm. Corp., Seoul, Korea) was performed for the antrum and the corpus to evaluate Helicobacter pylori infection. Blood sampling was performed to determined H. pylori lg G antibody titer using Immulite $2000 ®$ immunoassay system (Diagnostic Product Corporation, Los Angeles, CA, USA). H.pylori lg G titer was classified as negative $(<0.9 \mathrm{U} / \mathrm{m}, \mathrm{L})$, equivocal $(0.9-1.5 \mathrm{U} / \mathrm{mL})$ and positive ( $\geq 1.6 \mathrm{U} / \mathrm{mL}$ ). H.pylori status was defined as follows: No infection was defined as showing both negative H.pylori tests and had no history of H.pylori eradication. Past infection was defined as positive test H.pylori lg $\mathrm{G}$ and negative rapid urease test results or a history of H.pylori eradication and negative rapid urease test. Current $H$.pylori infection was defined a positive any of the test result without history of H.pylori eradication.

\section{Endoscopic Score}

Endoscopic scoring of gastric atrophy using WLE, NBI, and NFM was assessed and classified according to the Kimura-Takemoto classification: C0 (no atrophy) and C1 (mild) were scored as 0, C2 and C3 (moderate) as 1, and 0-1, 0-2, and 0-3 (severe) as 2 [28-30]. Endoscopic scoring for intestinal metaplasia was defined as at least one of the following findings under NBI-NFM: light blue crest (LBS, Figure 1A), white opaque substances (WOS, Figure 1B), and/or a tubular/granular pit pattern of the corpus (Figure 1C) [8]. LBS was a fine, blue-whitish line observed on the crests of the epithelial surface or gyri [10]. WOS was a white opaque substance appearing on part of the surface under the NBI-magnifying view [23]. Score 0 was none of the three findings suggestive of intestinal metaplasia. Score 1 was presence at least one finding on the antrum. Score 2 was the presence at least one finding on the corpus. If findings suggestive of intestinal metaplasia were observed $>1 / 2$ of the picture at the antrum, score 1 was added and if the findings were observed $>1 / 2$ of the picture at the corpus, score 2 was added. Therefore, the score for atrophic gastritis ranged from 0 to 2 and the score for intestinal metaplasia ranged from 0 to 4 .

\section{Histology}

Biopsy specimens were fixed and paraffin-embedded, and each section was stained with hematoxylin and eosin (H\&E). Atrophic gastritis and intestinal metaplasia were interpreted by an experienced gastrointestinal pathologist (PYS) who was unaware of the endoscopic scores, using the updated Sydney system. The presence of atrophy was assessed based on findings of shrinking or vanishing of the glands and fibrosis of the lamina propria. The presence of metaplasia was assessed based on findings of intestinal metaplasia or pseudo-metaplasia of the corpus. OLGA and OLGLIM staging were applied according to the OLGA and OLGIM guidelines [7, 31, 32]. In each of the two areas (the lesser curvature and greater curvature), an overall atrophy and metaplasia score expressed the sum of the percentages of atrophy/ metaplasia changes and was divided by two [31]. Atrophic gastritis was graded as no, mild (1- 
$30 \%)$, moderate $(31-60 \%)$ or severe $(>60 \%)$ atrophy of the observed biopsy tissue from the antrum and corpus, respectively [31]. Metaplasia was graded from no, mild (1-9), moderate (10-29), or severe metaplasia ( $\geq 30 \%$ ) at each antrum and corpus biopsy level, respectively. Stages $0,1,2$ were placed into the low-risk group whereas stage 3 and 4 were the high-risk group.

\section{Statistical analysis}

Continuous and categorical variables are presented as median (interquartile range) and number (\%), respectively. Correlations between the endoscopic score and histology were calculated using Spearman correlation analysis. Sensitivity analysis was performed to find the best correlation between the high-risk endoscopic score and the high-risk OLGA or OLGIM staging. The sensitivity, specificity, positive predictive value (PPV), and negative predictive value (NPV) for each cut-off value of the endoscopic scores were calculated. Receiver Operating Characteristic (ROC) curves and the area under the curve (AUCs) were calculated for binary classification. Logistic regression and odds ratios were used to assess the endoscopic risk group for high OLGA and OLGIM stages. Statistical analyses were performed using SAS (Statistical Analysis Software 9.4, SAS Institute Inc., Cary, North Carolina, USA), and P values $<0.05$ were considered as statistically significant.

\section{Results}

\section{Baseline characteristics}

The baseline characteristics of the patients are summarized in Table 1. Their median age was 59 years old and 58.8\% (30/51) were men. The reasons for undergoing EGD were as follows: abnormal findings from the local hospital $(25 / 51,49.0 \%)$, dyspepsia $(12 / 51,23.5 \%)$, screening $(6 / 51,11.8 \%)$, abdominal pain $(4 / 51,7.8 \%)$, chest pain $(3 / 51,5.9 \%)$ and reflux symptom $(1 / 51,2.0 \%)$. Ten patients $(19.6 \%)$ had a history of $H$. pylori eradication. H. pylori lgG was positive in 34 patients $(66.7 \%)$ and the rapid urease test was positive in 26 patients (51.0\%). 
Table 1

Baseline characteristics of the study population

\begin{tabular}{|ll|}
\hline Characteristics & Patients \\
\hline Age, years & $59(52-63)$ \\
\hline Male & $30(58.8)$ \\
\hline Body mass index $\left(\mathrm{kg} / \mathrm{m}^{2}\right)$ & $24.9 \pm 2.4 / 25.4(23.1-26.9)$ \\
\hline Smoking & \\
\hline Non-smoking & $29(56.9)$ \\
\hline Past smoker & $15(29.4)$ \\
\hline Current smoker & $7(13.7)$ \\
\hline Alcohol consumption & \\
\hline Non-drinker & $21(41.2)$ \\
\hline Past drinker & $4(7.8)$ \\
\hline Current drinker & $26(51.0)$ \\
\hline Current medication & $32(62.7)$ \\
\hline Acid suppressant & $10(19.6)$ \\
\hline Hypoglycemic agents & $6(11.8)$ \\
\hline Antihypertensive drugs & $11(21.6)$ \\
\hline Lipid lowering gents & $11(21.6)$ \\
\hline Acid suppressant & $10(19.6)$ \\
\hline Aspirin & $2(3.9)$ \\
\hline NSAlDs & $3(5.9)$ \\
\hline Reason for EGD & $25(49.0)$ \\
\hline Abnormal finding on outside endoscopy & \\
\hline Abdominal pain & \\
\hline
\end{tabular}

Values are presented as number (percent) or median (interquartile range).

${ }^{+}$The cut off value for H.pylori sero-positivity was defined as $\geq 1.6$ and

Sero-negativity was defined as the value was $<0.9$ 


\begin{tabular}{|ll|}
\hline Characteristics & Patients \\
\hline Dyspepsia & $12(23.5)$ \\
\hline Chest pain & $3(5.9)$ \\
\hline Screening & $6(11.8)$ \\
\hline Reflux & $1(2.0)$ \\
\hline Family history of gastric cancer & $11(21.6)$ \\
\hline History of peptic ulcer disease & $3(5.9)$ \\
\hline History of upper Gl bleeding & $0(0)$ \\
\hline History of endoscopic resection & $1(2.0)$ \\
\hline History of $H$. pylori eradication & $10(19.6)$ \\
\hline H. pylori lgG ${ }^{+}$ & \\
\hline Positive & $34(66.7)$ \\
\hline Titer & $4.5(2.5-6)$ \\
\hline Positive rapid urease test & $26(51.0)$ \\
\hline Laboratory findings & $79(71-89)$ \\
\hline WBC & $6.5(5.5-7.2)$ \\
\hline Hemoglobin & $14.2(13-15.4)$ \\
\hline Platelet & $233(211-269)$ \\
\hline Systolic blood pressure (mmHg) & $134-86)$ \\
\hline Diastolic blood pressure (mmHg) & $790)$ \\
\hline Pulse rate (/min) & or median (interquartile range). \\
\hline Values are presented as number (percent) & \\
\hline The cut off value for H.pylori sero-positivity was defined as $\geq 1.6$ and \\
\hline Sero-negativity was defined as the value was < 0.9 \\
\hline
\end{tabular}

Table 2. Distribution of risk groups of endoscopic score using NBI-NFM and OLGA and OLGIM staging 


\begin{tabular}{|clllll|}
\hline \multicolumn{5}{|c|}{ Endoscopic atrophy/metaplasia score } & \\
\cline { 3 - 5 } & & Low risk & High risk & Agreement & P value \\
\hline \multirow{2}{*}{ OLGA } & Low risk $(0-2)$ & $20(87.0 \%)$ & $7(25.0 \%)$ & $80.4 \%$ & $<0.001$ \\
\cline { 2 - 4 } & High risk $(3,4)$ & $3(13.0 \%)$ & $21(75.0 \%)$ & & \\
\cline { 1 - 4 } OLGIM & Low risk $(0-2)$ & $16(100 \%)$ & $11(31.4 \%)$ & $78.4 \%$ & $<0.001$ \\
\cline { 2 - 4 } & High risk $(3,4)$ & $0(0)$ & $24(68.6 \%)$ & & \\
\hline
\end{tabular}

\section{Endoscopic scores and histologic staging}

Endoscopic scores of the study population are shown in Figure 2a and 2b. As for the atrophy score, score 0 (C-0, and C-1) was assessed in 10 patients (19.6\%), score 1 (C-2, and C-3) in 13 patients (25.5\%), and score 2 (0-1, 0-2, and 0-3) in 28 patients (54.9\%). For the metaplasia score, score 0 was assessed in 13 patients (25.5\%), score 1 in 3 patients (5.9\%), score 2 in 24 patients (47.1\%), score 3 in 5 patients $(9.8 \%)$, and score 4 in 6 patients (11.8\%).

The OLGA and OLGIM scores are shown in Figure 2c and 2d. As for OLGA staging, stage 0 was found in 10 patients $(19.6 \%)$, stage I in 8 patients $(15.7 \%)$, stage II in 9 patients $(17.6 \%)$, stage III in 7 patients (13.7\%), and stage IV in 17 patients (33.3\%). Regarding OLGLIM staging, stage 0 was observed in 13 patients (25.5\%), stage I in 9 patients (17.6\%), stage II in 5 patients (9.8\%), stage III in 14 patients (27.5\%), and stage IV in 10 patients (19.6\%). Therefore, $47.0 \%$ of the patients were in the high-risk OLGA group (stage III or IV) and $47.1 \%$ of the patients were in the high-risk OLGIM group (stage III or IV).

Endoscopic scores and histologic staging according to H.pylori infection status are shown in Figure 3. Endoscopic scores for gastric atrophy and metaplasia were significantly higher in current H.pylori infected patients ( $p=0.002$ and 0.012 , respectively, Figure $3 a$ and $3 b$ ). OLGA and OLGIM staging were also significantly higher in current H.pylori infected patients $(p=0.002$, and 0.003 , respectively, Figure $3 c$ and $3 d)$.

\section{Correlation between endoscopic scores and histology}

The correlation coefficient for atrophy between the endoscopic and histologic scores was 0.70 ( $95 \% \mathrm{Cl}$ : $0.52-0.81, p<0.001)$ and for metaplasia it was $0.75(95 \% \mathrm{Cl}: 0.60-0.85 ; p<0.001)$. The distribution of risk groups using NBI-NFM and OLGA or OLGIM staging are shown in Table 2.

For atrophic gastritis, endoscopic score $>1$ correlated with OLGA Stage III and IV with a sensitivity, specificity, PPV, NPV, and accuracy of $88 \%, 74 \%, 75 \%, 87 \%$, and $80.4 \%$, respectively. On ROC curve analysis, the area under the curve for atrophy was 0.81 (Figure $4 a$ ) and the kappa value was 0.61 (95\% Cl: $0.4-0.82, p<0.001)$. 
For metaplasia, endoscopic score > 1 correlated with high OLGIM Stage III and IV with sensitivity, specificity, PPV, NPV, and accuracy of $100 \%, 59 \%, 69 \%, 100 \%$, and $78.4 \%$, respectively. On ROC curve analysis, the area under the curve was 0.87 (Figure 4b) and the kappa value was 0.58 (95\% Cl: 0.38-0.78, $p<0.001)$.

\section{Discussion}

In this study, we developed endoscopic scores evaluating the atrophy and metaplasia of gastric mucosa using NBI and NFM and assessed the correlation between the endoscopic scores and histology. Previous studies evaluating atrophy and intestinal metaplasia reported the usefulness of NBI and/or magnifying endoscopy, not diagnostic performance of NFM. Conventional magnifying endoscopy is time consuming for observation and can be performed only by experienced endoscopist. However, near focus function can be used with several additional minutes and easily applicable without special training. In our study, an atrophic border (F-line) could be readily observed by adding an NBI examination and typical findings of intestinal metaplasia (LBC, WOS, and tubular or granular pit of the corpus), which has been previously reported using NBI magnifying endoscopy, and it could also easily be seen with NBI with NFM. When applying endoscopic atrophy scores based on the Kimura-Takemoto classification and the endoscopic metaplasia score based on the typical NBI-magnification view of the metaplasia and the extent, score $>1$ was a high-risk group for both atrophy and metaplasia and it showed a strong positive correlation ( 0.70 for atrophy and 0.75 for metaplasia) with high-risk OLGA and OLGIM staging.

Discrepancies between endoscopic and histologic diagnosis of gastric atrophy and metaplasia have been reported. In a study reporting discrepancies between histological and endoscopic findings for atrophic gastritis in 1330 patients, the sensitivity and specificity of atrophic gastritis in the antrum were $61.5 \%$ and $57.7 \%$, and in the corpus were $46.8 \%$ and $76.4 \%$ [18]. As for intestinal metaplasia, they were 24.0 and $91.9 \%$ in the antrum and $24.2 . \%$ and $88 \%$ in the corpus [19]. In this study, the sensitivities for the diagnosis of endoscopic metaplasia were lower than our results because their diagnosis of metaplasia was evaluated only with white light endoscopy. In a recent multicenter validation study for endoscopic grading of gastric intestinal metaplasia, endoscopic score $>4$ correlated with stage III and IV OLGIM with $89.4 \%$ of sensitivity, $94.6 \%$ of specificity, $79.2 \%$ of PPV, and $97.5 \%$ of NPV [12]. In the study, the authors used white light endoscopy and NBI (without magnification) and the score ranged from 0 to 10 . Our result showed higher sensitivity and PPV whereas lower specificity and NPV than this European study. We assume that a score system with high sensitivity and PPV is more useful to screen gastric cancer in the countries with high prevalence of gastric cancer.

Endoscopic grading of atrophic gastritis according to the Kimura-Takemoto classification is widely used. This system significantly reflects both the extent and severity of gastric atrophy because the extent correlates with the degree of severity. Endoscopic atrophic score was graded as 0 (none, C0 or mild, C1), 1 (moderate, C2 and C3), and 2 (severe, 01, 02, and 03), and when the score was 2, the score correlated well with OLGA Stage III and IV in our result. Recent studies showed the risk of cancer differs according to 
this grading $[20,21]$. In a study evaluating 9378 subjects underwent cancer screening, C0-1, C2-3, and open type showed $0.10 \%, 0.16 \%$, and $0.31 \%$ of annual rate of gastric cancer occurrence, respectively [21].

H.pylori infection status was also significantly associated with not only high endoscopic atrophy/metaplasia score, but also high OLGA and OLGIM system. Our result is consistent with the date from the previous report evaluating OLGA and OLGIM system according to age and H.pylori status in the Korean population [22]. In the study, the proportion of high OLGA and OLGIM stages was significantly increased with H.pylori infection (OR=8.46) and high risk OLGA and OLGIM stages were uncommon in the H.pylori negative subjects.

We diagnosed endoscopic metaplasia showing at least one finding of LBS, WOS, and tubular/granular pit pattern of the corpus using NBI-NFM. Those findings have been reported as a useful marker for endoscopic diagnosis of intestinal metaplasia with $50-89 \%$ sensitivity and $80-100 \%$ specificity $[10,13$, 23]. However, these findings suggest only the presence of intestinal metaplasia and do not give information about the extent and the severity of intestinal metaplasia. Therefore, we added scores according to the site (antrum or corpus) and severity (whether the findings suggestive of intestinal metaplasia were observed in more than $1 / 2$ area of the picture or not) and our endoscopic metaplasia score showed a good correlation with histologic staging. In a cross-sectional study of 55 patients, the authors developed an endoscopic score using NBI magnifying endoscopy and they combined the scores for the antrum and the corpus mainly based on findings of intestinal metaplasia [8]. The degree of correspondence between the high-risk NBI-ME finding and a high histology score was $89.1 \%$, which was similar to our result.

Our endoscopic atrophic and metaplasia scores tend to overestimate the OLGA and OLGIM system. We speculate that it is because multifocal atrophy or metaplasia could be underestimated on histologic examination if biopsy samples were not taken from the exact site of the atrophy or metaplasia change. In addition, we omitted taking a biopsy sample from the incisura angularis, which could affect the histologic diagnosis. In a study assessing the value of incisura angularis biopsy, a general down-grading of stage by $4.0 \%$ for OLGIM and $30-35 \%$ downgrading for high-risk OLGA/OLGIM stages were observed if the incisura angularis was excluded from the biopsy [24].

The proportion of high-risk OLGA and OLGIM (stage III or IV) patents was high at $47 \%$. A previous study on the OLGA and OLGIM stage distribution in a Korean screening papulation, high-risk OLGA patients were only $16.6 \%$ and high-risk OLGIM, 9.5\% [22]. It is inferred that the differences came from the characteristics of our study population. Many of our patients (49\%) underwent diagnostic endoscopy because of abnormal EGD findings such as early gastric cancer or dysplasia at a local clinic. In a study evaluating OLGA and OLGIM stage in gastric cancer patients, the proportion of high-risk OLGA and OLGIM patients was similar to our study (46.2\% OLGA and 46.1\% OLGIM, respectively) [25]. Furthermore, the past history $(19.6 \%)$ or current infection rate $(51 \%)$ of $H$. pylori was relatively high in our population, and this may affect the high proportion of high-risk OLGA and OLGIM staging. 
It is known that OLGA staging reliably predicts the risk of developing gastric cancer. In a prospective study of 1755 patients followed up for 5 years, the risk for gastric dysplasia or cancer was null in patients stage 0,1 and III, while it was 36.5 per 1000 person-years among patients at stage 3 and 63.1 per 1000 person-years among patients at stage IV[26]. In a retrospective cohort study, OLGIM showed a better predictive value for gastric cancer development than OLGA and the standardized incidence rate for high risk OLGIM was 4.0[27]. OLGIM staging has an advantage of high inter-observer agreement, but a substantial proportion of high-risk patients would be missed if only OLGIM staging was applied[24]. Applying both OLGA and OLGIM staging is necessary for accurate prediction of gastric cancer risk. In the same vein, we infer that evaluations of both atrophic gastritis and intestinal metaplasia are necessary to screen high-risk patients for gastric cancer.

This study has several limitations. First, the number of enrolled patients was small. Second, we could not evaluate the inter-observer agreement of the pathologists. Third, we did not take a biopsy of the incisura angularis, which could result in down-grading of histology. However, our prospective study showed the feasibility of applying endoscopic scores using NBI and NFM, and the high-risk scores correlate well with high OLGA and OLGIM staging. Further studies applying these endoscopic scores for large screening populations and evaluating the actual incidence of gastric cancer during long-term follow-up of high-risk patients are needed.

In conclusion, we developed an endoscopic score for gastric atrophy and intestinal metaplasia using NBINFM, and the endoscopic scores showed a strong correlation with OLGA/OLGIM staging. Thus, it could be a practical alternative to taking multiple tissue biopsies.

\section{Declarations}

\section{Ethics approval and consent to participate}

This study was performed following the Helsinki Declaration of the World Medical Association. This study was approved by the Ethics Committee of Asan Medical Center (number: 2017-0947) and was registered with a clinical research information service (http://cris.nih.go.kr, KCT0004377). Written informed consent was obtained from all the participants.

\section{Consent for publication}

Not applicable

\section{Availability of data and materials}

The datasets generated and/or analysed during the current study are available for the corresponding author on reasonable request.

\section{Competing interests}

The authors have declared that they have no competing interests.

\section{Funding}

Page $12 / 18$ 
This study was supported by the Korean College of Helicobacter and an Upper Gastrointestinal Research Foundation grant (number: 2017).

\section{Authors' contributions}

This study was designed, directed and coordinated by CKD, PYS, and NHK. CKD and NHK carried out recruitment of the patients and their endoscopies. Pathologic review was performed by PYS. KHJ performed the statistical analysis. CKD and NHK participated data interpretation and drafted the manuscript. All authors reviewed the manuscript and approved the final version of the manuscript.

\section{Acknowledgements}

Not applicable

\section{References}

1. Correa P, Piazuelo MB. The gastric precancerous cascade. J Dig Dis. 2012;13:2-9.

2. de Vries AC, van Grieken NC, Looman CW, Casparie MK, de Vries E, Meijer GA, et al. Gastric cancer risk in patients with premalignant gastric lesions: a nationwide cohort study in the Netherlands. Gastroenterology. 2008;134:945-52.

3. Cassaro M, Rugge M, Gutierrez O, Leandro G, Graham DY, Genta RM. Topographic patterns of intestinal metaplasia and gastric cancer. Am J Gastroenterol. 2000;95:1431-8.

4. Shichijo S, Hirata Y, Niikura R, Hayakawa Y, Yamada A, Ushiku T, et al. Histologic intestinal metaplasia and endoscopic atrophy are predictors of gastric cancer development after Helicobacter pylori eradication. Gastrointest Endosc. 2016;84:618-24.

5. Sakitani K, Hirata Y, Watabe H, Yamada A, Sugimoto T, Yamaji Y, et al. Gastric cancer risk according to the distribution of intestinal metaplasia and neutrophil infiltration. J Gastroenterol Hepatol. 2011;26:1570-5.

6. Rugge M, Genta RM. Staging and grading of chronic gastritis. Hum Pathol. 2005;36:228-33.

7. Capelle LG, de Vries AC, Haringsma J, Ter Borg F, de Vries RA, Bruno MJ, et al. The staging of gastritis with the OLGA system by using intestinal metaplasia as an accurate alternative for atrophic gastritis. Gastrointest Endosc. 2010;71:1150-8.

8. Saka A, Yagi K, Nimura S. OLGA- and OLGIM-based staging of gastritis using narrow-band imaging magnifying endoscopy. Dig Endosc. 2015;27:735-42.

9. Kawamura M, Abe S, Oikawa K, Terai S, Saito M, Shibuya D, et al. Topographic differences in gastric micromucosal patterns observed by magnifying endoscopy with narrow band imaging. $\mathrm{J}$ Gastroenterol Hepatol. 2011;26:477-83.

10. Uedo N, Ishihara R, lishi H, Yamamoto S, Yamamoto S, Yamada T, et al. A new method of diagnosing gastric intestinal metaplasia: narrow-band imaging with magnifying endoscopy. Endoscopy. 2006;38:819-24. 
11. Bansal A, Ulusarac $O$, Mathur S, Sharma P. Correlation between narrow band imaging and nonneoplastic gastric pathology: a pilot feasibility trial. Gastrointest Endosc. 2008;67:210-6.

12. Esposito G, Pimentel-Nunes P, Angeletti S, Castro R, Libânio D, Galli G, et al. Endoscopic grading of gastric intestinal metaplasia (EGGIM): a multicenter validation study. Endoscopy. 2019;51:515-21.

13. Savarino E, Corbo M, Dulbecco P, Gemignani L, Giambruno E, Mastracci L, et al. Narrow-band imaging with magnifying endoscopy is accurate for detecting gastric intestinal metaplasia. World $\mathrm{J}$ Gastroenterol. 2013;19:2668-75.

14. Ikematsu H, Matsuda T, Osera S, Imajoh M, Kadota T, Morimoto H, et al. Usefulness of narrow-band imaging with dual-focus magnification for differential diagnosis of small colorectal polyps. Surg Endosc. 2015;29:844-50.

15. Ciocâlteu AM, CârŢână ET, Florescu DN, Gheonea IA, Troponete IM, Ciurea T, et al. Narrow band imaging with near-focus mode for colorectal polyps' characterization. Rom J Morphol Embryol. 2016;57(2 Suppl):619-26.

16. Singh R, Jayanna M, Navadgi S, Ruszkiewicz A, Saito Y, Uedo N. Narrow-band imaging with dual focus magnification in differentiating colorectal neoplasia. Dig Endosc. 2013;25(Suppl 2):16-20.

17. Szura M, Pasternak A, Bucki K, Urbańczyk K, Matyja A. Two-stage optical system for colorectal polyp assessments. Surg Endosc. 2016;30:204-14.

18. Eshmuratov A, Nah JC, Kim N, Lee HS, Lee HE, Lee BH, et al. The correlation of endoscopic and histological diagnosis of gastric atrophy. Dig Dis Sci. 2010;55:1364-75.

19. Lim JH, Kim N, Lee HS, Choe G, Jo SY, Chon I, et al. Correlation between endoscopic and histological diagnoses of gastric intestinal metaplasia. Gut Liver. 2013;7:41-50.

20. Masuyama H, Yoshitake N, Sasai T, Nakamura T, Masuyama A, Zuiki T, et al. Relationship between the degree of endoscopic atrophy of the gastric mucosa and carcinogenic risk. Digestion. 2015;91:30-6.

21. Kaji K, Hashiba A, Uotani C, Yamaguchi Y, Ueno T, Ohno K, et al. Grading of atrophic gastritis is useful for risk stratification in endoscopic screening for gastric cancer. Am J Gastroenterol. 2019;114:71-9.

22. Nam JH, Choi IJ, Kook MC, Lee JY, Cho SJ, Nam SY, et al. OLGA and OLGIM stage distribution according to age and Helicobacter pylori status in the Korean population. Helicobacter. 2014;19:819.

23. Yao K, Iwashita A, Nambu M, Tanabe H, Nagahama T, Maki S, et al. Nature of white opaque substance in gastric epithelial neoplasia as visualized by magnifying endoscopy with narrow-band imaging. Dig Endosc. 2012;24:419-25.

24. Isajevs S, Liepniece-Karele I, Janciauskas D, Moisejevs G, Funka K, Kikuste I, et al. The effect of incisura angularis biopsy sampling on the assessment of gastritis stage. Eur $\mathrm{J}$ Gastroenterol Hepatol. 2014;26:510-3.

25. Cho SJ, Choi IJ, Kook MC, Nam BH, Kim CG, Lee JY, et al. Staging of intestinal- and diffuse-type gastric cancers with the OLGA and OLGIM staging systems. Aliment Pharmacol Ther. 2013;38:1292302. 
26. Rugge $M$, Meggio A, Pravadelli C, Barbareschi M, Fassan M, Gentilini M, et al. Gastritis staging in the endoscopic follow-up for the secondary prevention of gastric cancer: a 5-year prospective study of 1755 patients. Gut. 2019;68:11-7.

27. Nieminen AA, Kontto J, Puolakkainen P, Virtamo J, Kokkola A. Comparison of operative link for gastritis assessment, operative link on gastric intestinal metaplasia assessment, and TAIM stagings among men with atrophic gastritis. World J Gastroenterol. 2020;26:3447-57.

28. Kishino M, Nakamura S, Shiratori K. Clinical and endoscopic features of undifferentiated gastric cancer in patients with severe atrophic gastritis. Intern Med. 2016;55:857-62.

29. Sugimoto M, Ban H, Ichikawa H, Sahara S, Otsuka T, Inatomi O, et al. Efficacy of the Kyoto classification of gastritis in identifying patients at high risk for gastric cancer. Intern Med. 2017;56:579-86.

30. Miwata T, Quach DT, Hiyama T, Aoki R, Le HM, Tran PL, et al. Interobserver and intraobserver agreement for gastric mucosa atrophy. BMC Gastroenterol. 2015; doi:10.1186/s12876-015-0327-x.

31. Rugge M, Correa P, Di Mario F, El-Omar E, Fiocca R, Geboes K, et al. OLGA staging for gastritis: a tutorial. Dig Liver Dis. 2008;40:650-8.

32. Mescoli C, Gallo Lopez A, Taxa Rojas L, Jove Oblitas W, Fassan M, Rugge M. Gastritis staging as a clinical priority. Eur J Gastroenterol Hepatol. 2018;30:125-9.

\section{Figures}
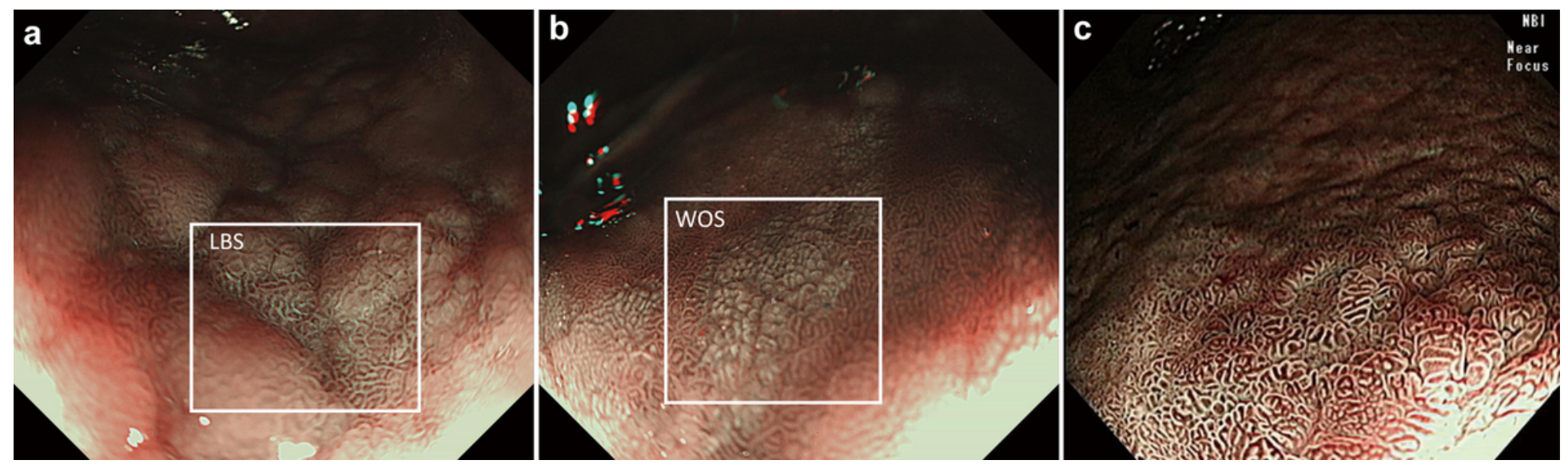

Figure 1

Representative endoscopic images using narrow band imaging with near focus mode A, Light blue crest (LBS) in antrum (box). B, White opaque substances (WOC) in antrum (box). C, Tubular or granular pit pattern in corpus. 
a

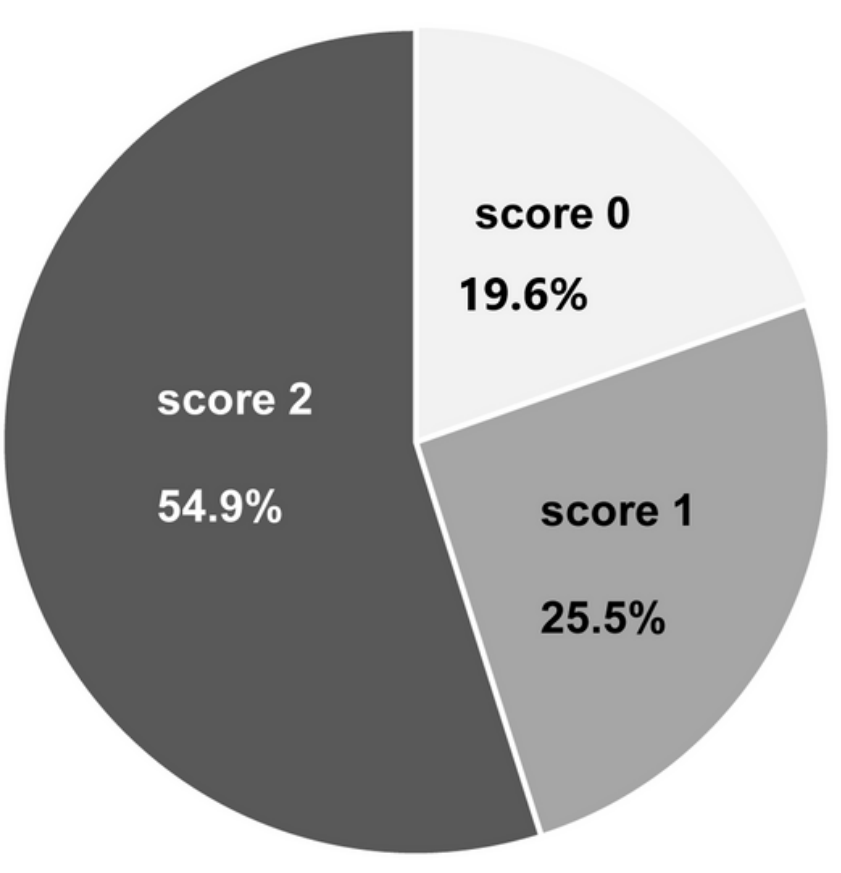

C

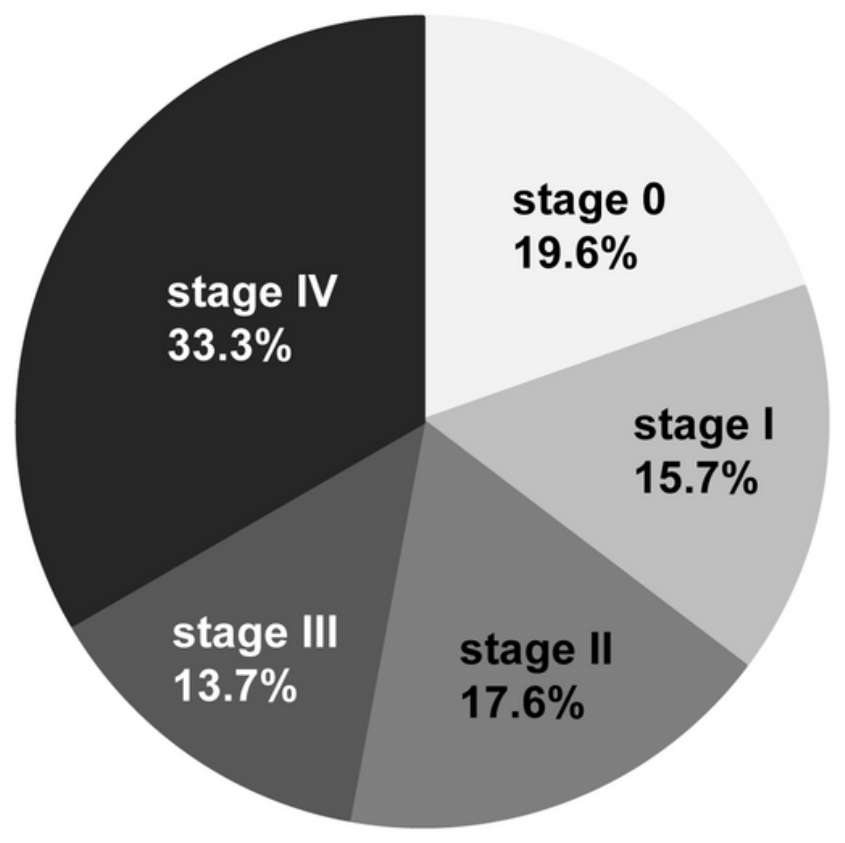

b

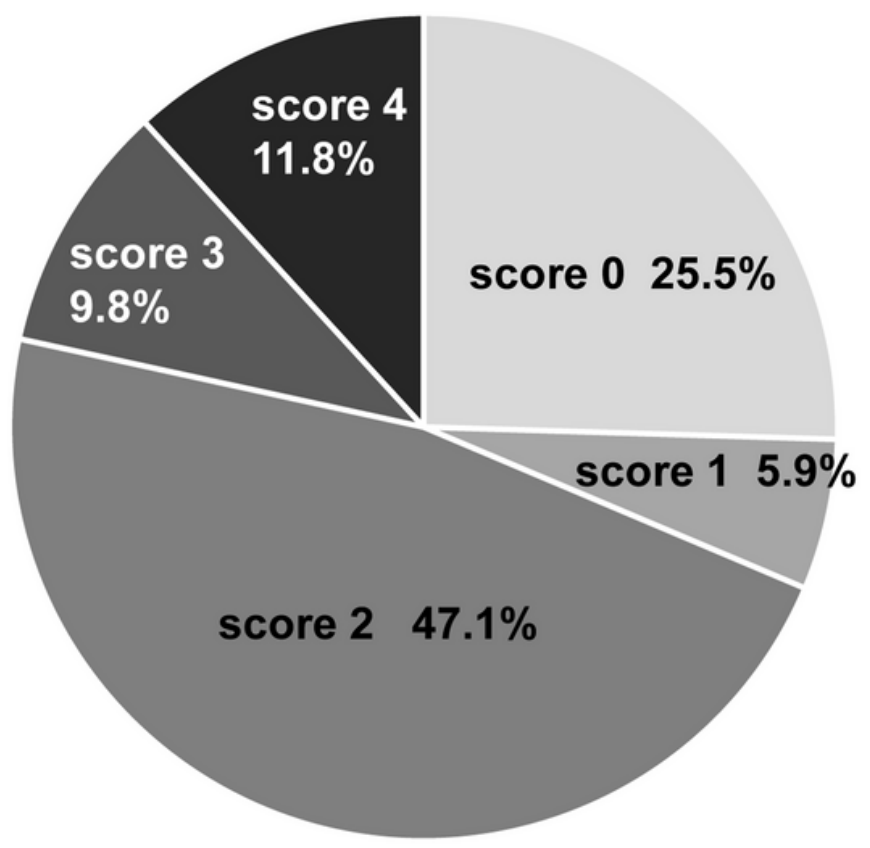

d

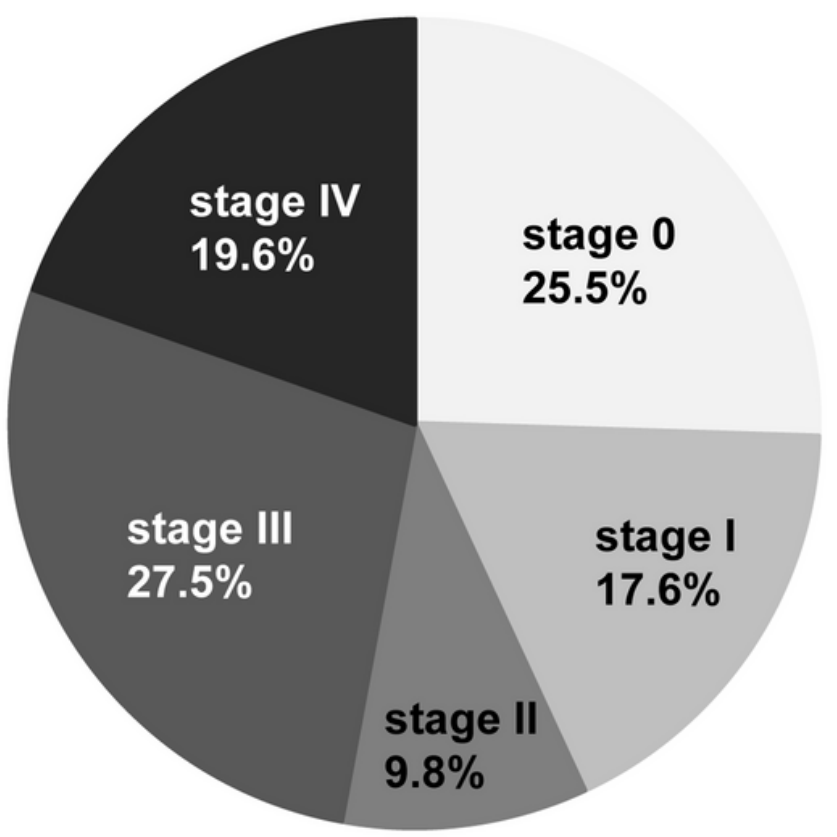

Figure 2

Endoscopic and histologic scores of the study population A, Endoscopic scores for gastric atrophy. B. Endoscopic score for intestinal metaplasia. C, OLGA stage. D, OLGIM stage. 
a

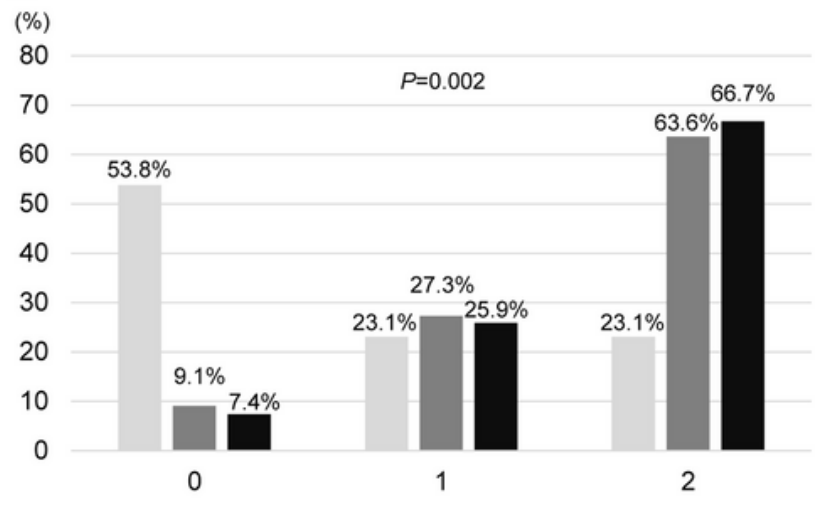

No HP infection $\approx$ past $\mathrm{Hp}$ infection $\quad$-Current $\mathrm{Hp}$ infection

\section{b}

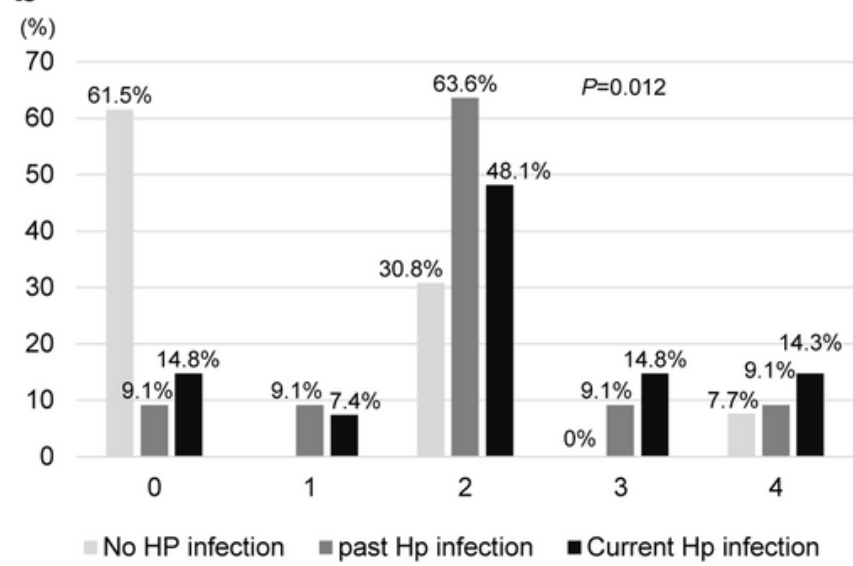

C

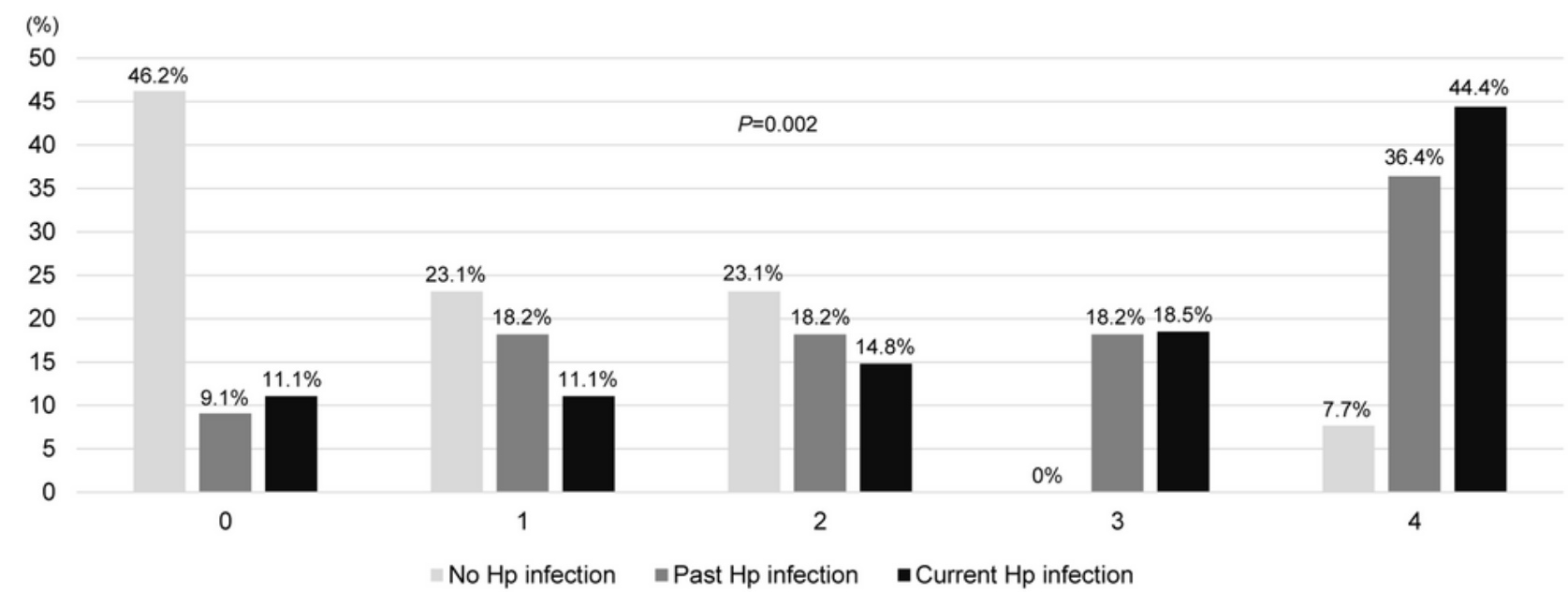

d

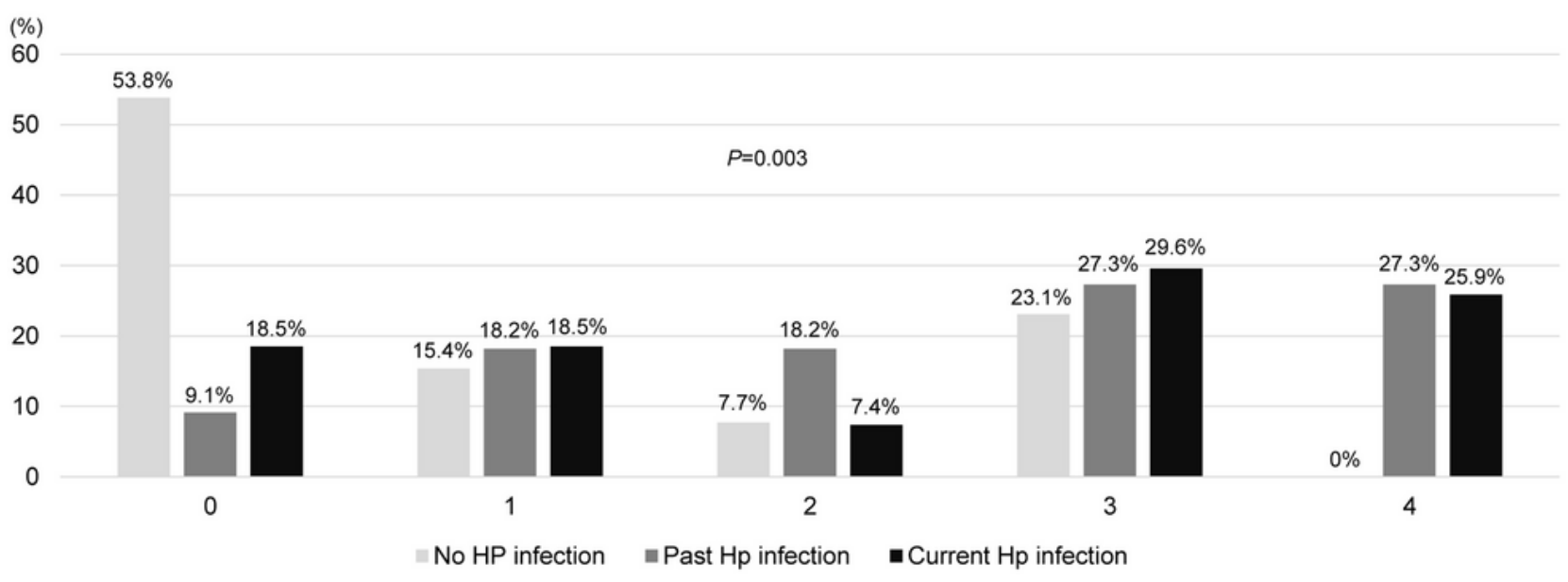

Figure 3

Endoscopic and histologic scores according to H.pylori status A. Endoscopic score for gastric atrophy. B, Endoscopic score for metaplasia. C, OLGA stage D, OLGIM stage 

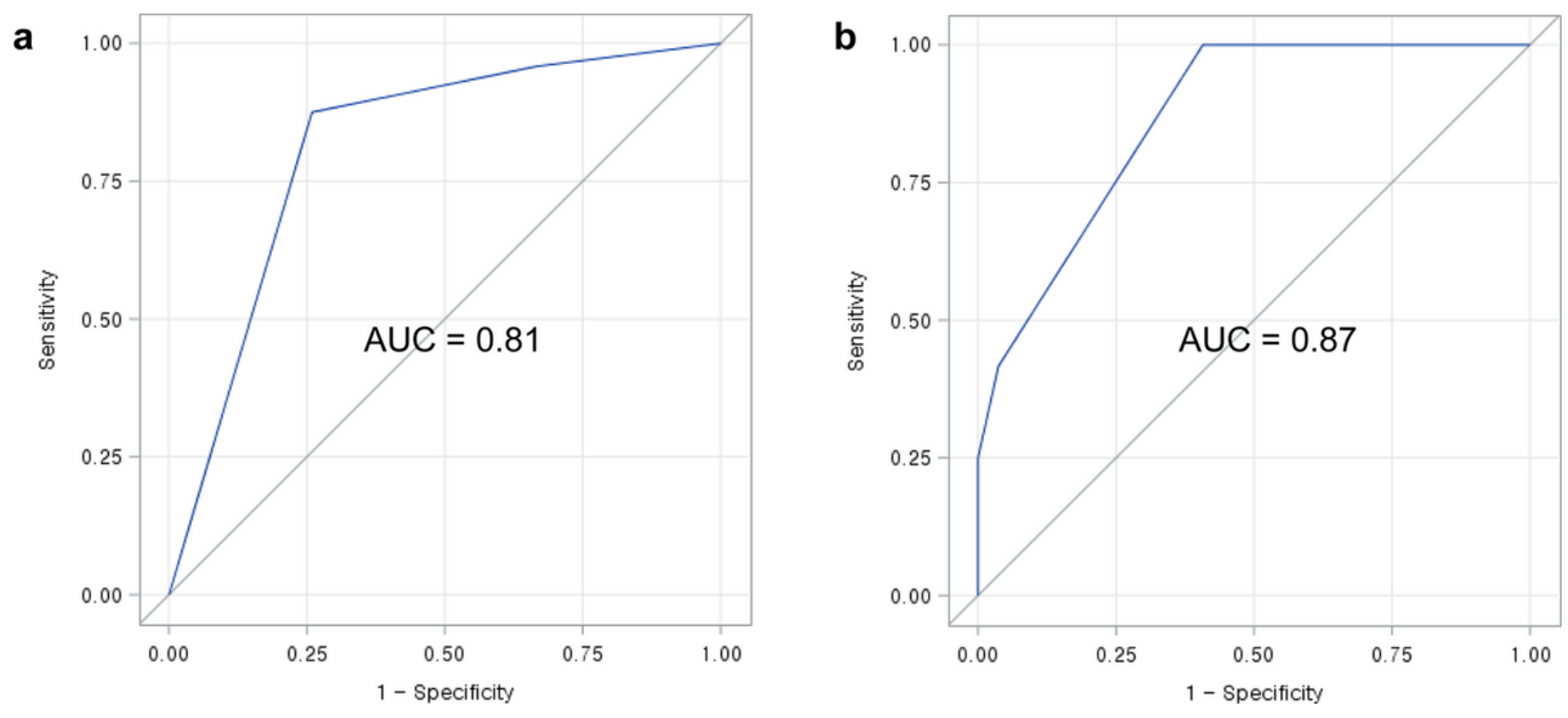

Figure 4

Correlation between endoscopic score and histologic stage (OLGA and OLGIM stage) 